\title{
Safety of nintedanib added to pirfenidone treatment for idiopathic pulmonary fibrosis
}

\author{
Kevin R. Flaherty ${ }^{1}$, Charlene D. Fell ${ }^{2}$, J. Terrill Huggins ${ }^{3}$, Hilario Nunes ${ }^{4}$, \\ Robert Sussman ${ }^{5}$, Claudia Valenzuela ${ }^{6}$, Ute Petzinger ${ }^{7}$, John L. Stauffer ${ }^{8}$, \\ Frank Gilberg ${ }^{9}$, Monica Bengus ${ }^{9}$ and Marlies Wijsenbeek ${ }^{10}$
}

\begin{abstract}
Affiliations: ${ }^{1}$ Division of Pulmonary and Critical Care Medicine, University of Michigan, Ann Arbor, MI, USA. ${ }^{2}$ Division of Respirology, University of Calgary, Calgary, AB, Canada. ${ }^{3}$ Division of Pulmonary, Critical Care, Allergy and Sleep Medicine, Medical University of South Carolina, Charleston, SC, USA. ${ }^{4}$ Dept of Respiratory Medicine, EA2363, Avicenne University Hospital, Paris, France. ${ }^{5}$ Atlantic Health System, Overlook Medical Center, Summit, NJ, USA. 'Servicio de Neumología, Hospital Universitario de La Princesa, Instituto de Investigación Princesa, Madrid, Spain. ${ }^{7}$ Clinipace-Accovion GmbH, Eschborn, Germany. ${ }^{8}$ Genentech, Inc., South San Francisco, CA, USA. ${ }^{9}$ F. Hoffmann-La Roche, Ltd, Basel, Switzerland. ${ }^{10}$ Dept of Respiratory Medicine, Erasmus University Medical Center, Rotterdam, The Netherlands.
\end{abstract}

Correspondence: Kevin R. Flaherty, Division of Pulmonary and Critical Care Medicine, University of Michigan, 3916 Taubman Center, 1500 East Medical Center Drive, Ann Arbor, MI 48109, USA.

E-mail: flahertyamed.umich.edu

@ERSpublications

Combined pirfenidone and nintedanib was tolerated by the majority of patients with IPF, encouraging further study http://ow.ly/1Iq030kaZuD

Cite this article as: Flaherty KR, Fell CD, Huggins JT, et al. Safety of nintedanib added to pirfenidone treatment for idiopathic pulmonary fibrosis. Eur Respir J 2018; 52: 1800230 [https://doi.org/10.1183/ 13993003.00230-2018].

ABSTRACT We assessed safety and tolerability of treatment with pirfenidone (1602-2403 mg.day $\left.{ }^{-1}\right)$ and nintedanib (200-300 $\left.\mathrm{mg} \cdot \mathrm{day}^{-1}\right)$ in patients with idiopathic pulmonary fibrosis (IPF).

This 24-week, single-arm, open-label, phase IV study (ClinicalTrials.gov identifier NCT02598193) enrolled patients with IPF with forced vital capacity $\%$ pred $\geqslant 50 \%$ and diffusing capacity of the lung for carbon monoxide \% pred $\geqslant 30 \%$. Before initiating nintedanib, patients had received pirfenidone for

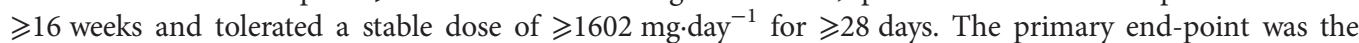
proportion of patients who completed 24 weeks of combination treatment on pirfenidone (1602$2403 \mathrm{mg} \cdot \mathrm{day}^{-1}$ ) and nintedanib (200-300 mg.day $\left.{ }^{-1}\right)$. Investigators recorded treatment-emergent adverse events (TEAEs), attributing them to pirfenidone, nintedanib, both or neither.

89 patients were enrolled; 73 completed 24 weeks of treatment (69 meeting the primary end-point) and 16 discontinued treatment prematurely (13 due to TEAEs). 74 patients had 418 treatment-related TEAEs, of which diarrhoea, nausea and vomiting were the most common. Two patients had serious treatmentrelated TEAEs.

Combined pirfenidone and nintedanib use for 24 weeks was tolerated by the majority of patients with IPF and associated with a similar pattern of TEAEs expected for either treatment alone. These results encourage further study of combination treatment with pirfenidone and nintedanib in patients with IPF.

This article has been revised according to the correction published in the October 2018 issue of the European Respiratory Journal.

Received: Feb 012018 | Accepted after revision: May 162018

This study is registered at ClinicalTrials.gov with identifier number NCT02598193.

Copyright CERS 2018. This ERJ Open article is open access and distributed under the terms of the Creative Commons Attribution Non-commercial Licence 4.0. 


\section{Introduction}

Idiopathic pulmonary fibrosis (IPF) is a progressive, irreversible, fatal, fibrosing lung disease of unknown cause [1, 2], with a survival rate lower than that reported for many common cancer types [1, 3-5]. Pirfenidone and nintedanib are approved as monotherapies for treatment of IPF [6, 7], and both received a conditional recommendation for use in the 2015 update to the American Thoracic Society (ATS)/European Respiratory Society (ERS)/Japanese Respiratory Society (JRS)/Latin American Thoracic Association (ALAT) clinical practice guideline [8]. Although pirfenidone and nintedanib have both demonstrated efficacy in reducing rates of disease progression compared with placebo, the disease is neither stopped nor reversed and patients continue to experience lung function decline while on treatment [9-11].

The differing putative mechanisms of action of pirfenidone and nintedanib [12-14] provide a physiological rationale for combining these two agents in an attempt to further reduce lung function decline in patients with IPF [15]. Even though both agents target the fibrotic cascade, decreasing fibroblast and myofibroblast production, and accumulation of extracellular matrix [12-14, 16, 17], evidence suggests that they may target distinct aspects of the fibrotic cascade. Although its mechanism of action has not been fully established, pirfenidone has been shown to act on multiple targets in vitro, including transforming growth factor- $\beta$-triggered events, mediated through glioma-associated oncogene homolog $2[12,13]$. Nintedanib is a tyrosine kinase inhibitor that blocks intracellular signalling of platelet-derived growth factor receptors, fibroblast growth factor receptors and vascular endothelial growth factor receptor [14].

Pirfenidone and nintedanib are both associated with gastrointestinal adverse events (AEs), with pirfenidone mainly associated with nausea and nintedanib mainly associated with diarrhoea $[9,10,18]$. A small Japanese randomised, double-blind, phase II dose-escalation trial of nintedanib $\left(100-300 \mathrm{mg}^{-\mathrm{day}^{-1}}\right.$ )

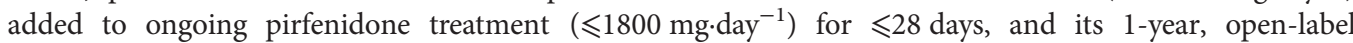
extension, indicated that safety and tolerability of combination treatment in patients with IPF was in line with AE profiles for each drug alone [19, 20]. Similarly, the recent INJOURNEY trial, an open-label, randomised trial of pirfenidone $\left(\leqslant 2403 \mathrm{mg} \cdot\right.$ day $\left.^{-1}\right)$ added to nintedanib treatment $\left(\leqslant 300 \mathrm{mg}^{- \text {day }^{-1}}\right)$ for 12 weeks, also found that the safety and tolerability profile of combination treatment was comparable with that of the individual drugs [21]. However, there are limited data on long-term safety of combination treatment. Here, we report results from a 24-week study investigating safety and tolerability of adding nintedanib to stable pirfenidone treatment in patients with IPF.

\section{Methods}

\section{Patients}

Eligible patients were aged 40-80 years at start of screening, with IPF based on the ATS/ERS/JRS/ALAT 2011 guideline [2]. Patients had received pirfenidone for $\geqslant 16$ weeks and tolerated a stable dose of 1602 $2403 \mathrm{mg} \cdot \mathrm{day}^{-1}$ for $\geqslant 28$ days without experiencing any moderate or severe adverse reactions considered by the investigator to be related to pirfenidone, or a pirfenidone treatment interruption for $>7$ days for any reason. Patients also had forced vital capacity (FVC) $\%$ pred $\geqslant 50 \%$ and diffusing capacity of the lung for carbon monoxide (DLCO) \% pred $\geqslant 30 \%$ at screening. Informed consent was obtained from each patient before any study activity or treatment was undertaken. Other inclusion and exclusion criteria are listed in supplementary table S1.

\section{Study design}

This was an international, single-arm, open-label, phase IV study (ClinicalTrials.gov identifier NCT02598193) that assessed safety and tolerability of 24 weeks of treatment with pirfenidone (1602$2403 \mathrm{mg} \cdot \mathrm{day}^{-1}$ ) and nintedanib (200-300 mg.day ${ }^{-1}$ ) in patients with IPF (figure 1). Patients started nintedanib treatment that was added to stable pirfenidone treatment on day 1 of the study. Combination treatment administration is further described in the supplementary methods .

Medications prohibited during the study are described in supplementary table S2.

\section{Assessments}

The primary objective was to investigate safety and tolerability of adding nintedanib to stable pirfenidone treatment in patients with IPF. This was assessed with the following end-points. 1) Primary end-point: proportion of patients who completed 24 weeks of combination treatment on pirfenidone (1602$2403 \mathrm{mg} \mathrm{day}^{-1}$ ) and nintedanib (200-300 mg.day $\left.{ }^{-1}\right)$. 2) Secondary end-points: a) proportion of patients who discontinued pirfenidone, nintedanib or both treatments before week 24 due to treatment-emergent AEs (TEAEs; AEs occurring after initiation of combination treatment until 28 days after the last dose of pirfenidone and nintedanib), b) total patient days of combination treatment, c) total duration in days from initiation of combination treatment to discontinuation of pirfenidone, nintedanib or both treatments, and d) frequency and timing of TEAEs and serious TEAEs. Changes from baseline in FVC, DLCO and King's 


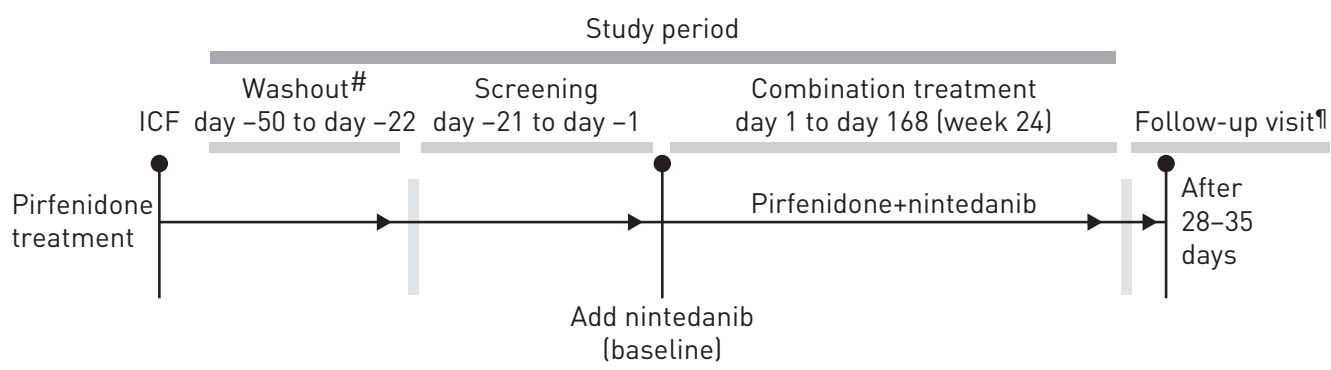

FIGURE 1 Study design. ICF: informed consent form. * : patients receiving prohibited medication discontinued that medication during washout (other patients entered directly into screening); ${ }^{\text {? }}$ : taking place 28-35 days after the end of combination treatment for patients who completed the 24-week combination treatment period, or 28-35 days after the decision to discontinue nintedanib for patients who discontinued prematurely or had treatment interrupted for $\geqslant 28$ consecutive days.

Brief Interstitial Lung Disease (K-BILD) questionnaire [22] score were assessed as exploratory end-points. Further details on study assessments are described in the supplementary methods.

\section{Statistical analysis}

This study planned to enrol 80 patients based on the sample size prediction described in the supplementary methods.

The safety population was defined as the population of all patients enrolled who received at least one dose of pirfenidone or nintedanib at baseline. Percentages were calculated based on number of patients with nonmissing data. Confidence intervals were based on a binomial distribution. This was a safety and tolerability study, with only one treatment group, which was not designed to assess efficacy of combination treatment. Thus, no formal statistical hypotheses were assessed and analyses were limited to descriptive statistics with no adjustments for multiplicity of end-points or within-subgroup comparisons.

\section{Results}

In total, 89 patients were enrolled from 36 centres in Canada, Denmark, France, Germany, Italy, the Netherlands, Spain and the USA between January and November 2016. Baseline patient demographics and

\section{TABLE 1 Demographic and baseline characteristics (safety population)}

\begin{tabular}{|c|c|}
\hline Patients & 89 \\
\hline Age years & $68.2 \pm 6.8$ \\
\hline Male & $71(80)$ \\
\hline \multicolumn{2}{|l|}{ Race } \\
\hline White & $84(94)$ \\
\hline Black or African-American & 3 (3) \\
\hline Other $\#$ & $2(2)$ \\
\hline Weight kg & $84.9 \pm 15.5$ \\
\hline Body mass index $\mathrm{kg} \cdot \mathrm{m}^{-2}$ & $28.6 \pm 4.6$ \\
\hline Previous tobacco use & $61(69)$ \\
\hline Duration of previous pirfenidone treatment months & $20.4 \pm 12.3$ \\
\hline \multicolumn{2}{|l|}{ FVC $\%$ pred mean $\pm S E$} \\
\hline Historical value closest to 6 months prior to screening" & $72.6 \pm 1.7$ \\
\hline At baseline & $71.8 \pm 1.7$ \\
\hline \multicolumn{2}{|l|}{ Patients categorised by baseline FVC $\%$ pred } \\
\hline$<65 \%$ & $32(36)$ \\
\hline $65-<80 \%$ & $31(35)$ \\
\hline$\geqslant 80 \%$ & $26(29)$ \\
\hline \multicolumn{2}{|l|}{ D.co $\%$ pred mean \pm SE } \\
\hline Historical value closest to 6 months prior to screening ${ }^{+}$ & $50.0 \pm 1.4$ \\
\hline At baseline & $48.4 \pm 1.3$ \\
\hline
\end{tabular}

Data are presented as $\mathrm{n}$, mean \pm SD or $\mathrm{n}(\%)$, unless otherwise stated. FVC: forced vital capacity; $D \mathrm{LCO}$ : diffusing capacity of the lung for carbon monoxide. ": includes Asian and mixed Asian/White; ": mean \pm SD time from assessment of historical value to screening was $3.0 \pm 1.9$ months ( $n=87) ;{ }^{+}$: mean \pm SD time from assessment of historical value to screening was $3.3 \pm 2.6$ months ( $n=87$ ). 
characteristics are shown in table 1. Patients had a mean \pm SE FVC $\%$ pred $71.8 \pm 1.7 \%$ and DLCO \% pred $48.4 \pm 1.3 \%$ at baseline (table 1). Measurements of FVC and DLCO closest to 6 months before start of screening were mean \pm SE $72.6 \pm 1.7 \%$ and $50.0 \pm 1.4 \%$ (table 1 ).

The most commonly reported medical history preferred terms ( $>10 \%$ of patients) were: gastro-oesophageal reflux disease (49 patients (55\%)), hypertension (44 patients (49\%)), hyperlipidaemia (20 patients $(23 \%)$ ), cough (18 patients $(20 \%)$ ), seasonal allergy (17 patients $(19 \%))$, IPF (16 patients (18\%); entering IPF as medical history was optional), sleep apnoea syndrome (16 patients (18\%)), hypercholesterolaemia (14 patients (16\%)), diabetes mellitus (13 patients (15\%)), osteoarthritis (12 patients (14\%)), allergic rhinitis (11 patients (12\%)), hypothyroidism (11 patients $(12 \%))$, depression (10 patients (11\%)), anxiety (nine patients $(10 \%)$ ), insomnia (nine patients $(10 \%)$ ), benign prostatic hyperplasia (nine patients $(10 \%)$ ) and obesity (nine patients (10\%)).

The most commonly reported concomitant medications ( $>25 \%$ of patients) were: proton pump inhibitors (66 patients $(74 \%)$ ), bronchodilators and antiasthmatics (e.g. salbutamol, ipratropium and budesonide; 52 patients (58\%)), statins (41 patients (46\%)), vitamins and minerals (41 patients (46\%)), steroids (38 patients $(43 \%))$, salicylates (35 patients $(39 \%)$ ), antidiarrhoeals (28 patients $(32 \%)$ ), antihistamines (26 patients $(29 \%))$, herbal, homeopathic and dietary supplements (24 patients $(27 \%)$ ), and nonsteroidal anti-inflammatory drugs (23 patients $(26 \%))$.

73 out of 89 (82\%, 95\% CI 72.5-89.4\%) patients completed 24 weeks of treatment plus follow-up and 69 out of 89 patients $(78 \%, 95 \%$ CI $67.4-85.7 \%)$ met the primary end-point (completion of 24 weeks of combination treatment at doses of pirfenidone $1602-2403 \mathrm{mg} \cdot \mathrm{day}^{-1}$ and nintedanib 200-300 mg.day ${ }^{-1}$ ) (figure 2). For the 16 patients who discontinued treatment prematurely, 13 patients discontinued due to TEAEs, one patient withdrew herself from the study and two patients discontinued for other reasons (one patient did not want to continue nintedanib and the other was listed for a lung transplant) (figure 2).

The mean \pm SD daily doses taken during combination treatment were pirfenidone $2339.3 \pm 183.7 \mathrm{mg} \cdot \mathrm{day}^{-1}$ and nintedanib $255.4 \pm 43.7 \mathrm{mg} \cdot \mathrm{day}^{-1}$ (including nintedanib titration period doses). Although 78 patients $(88 \%)$ received the target dose of $>1602 \mathrm{mg} \cdot \mathrm{day}^{-1}$ pirfenidone and $>200 \mathrm{mg} \cdot \mathrm{day}^{-1}$ nintedanib, 61 patients (69\%) received less than the full dose of pirfenidone $\left(2403 \mathrm{mg} \cdot \mathrm{day}^{-1}\right)$ and/or nintedanib $\left(287 \mathrm{mg} \cdot \mathrm{day}^{-1}\right.$ when including nintedanib titration period doses) overall during the study; the majority of patients did so

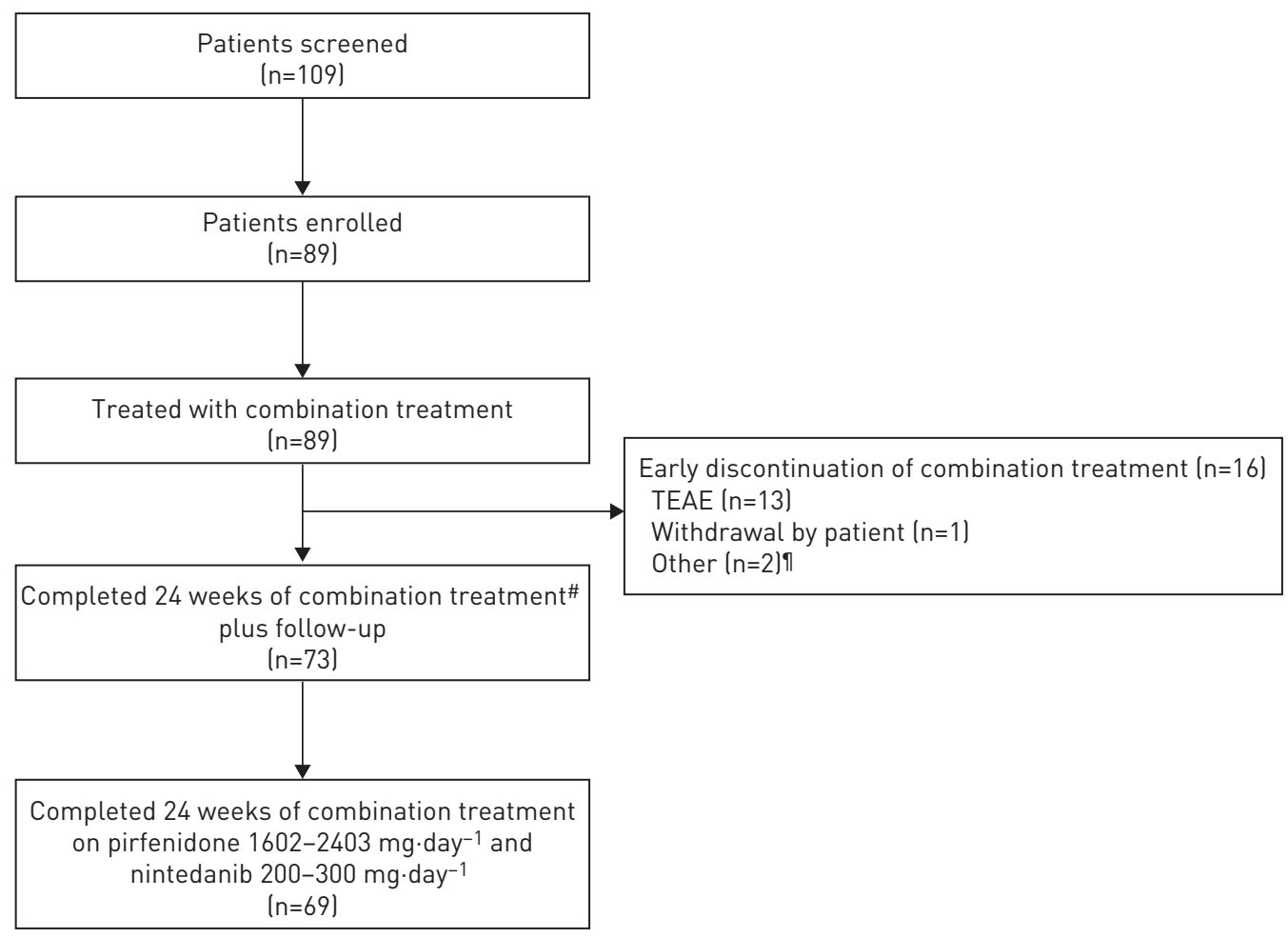

FIGURE 2 Patient disposition. TEAE: treatment-emergent adverse event. \#: combined pirfenidone and nintedanib taken at any dose; ${ }^{\text {ๆ }}$ : one patient was listed in the active lung transplant list and one patient did not wish to take nintedanib. 
because of temporary dose reductions and/or interruptions following TEAEs. The total number of patient days of combination treatment with pirfenidone and nintedanib was 13304 days, excluding dose interruptions (the expected patient days on treatment being 14952 days). Mean \pm sD duration of treatment during the study period was $21.3 \pm 6.3,20.8 \pm 6.2$ and $21.4 \pm 6.3$ weeks for pirfenidone, nintedanib and combination treatment, respectively, excluding dose interruptions. As the exposure analysis considered treatments administered from the start of combination treatment until study drug completion/early discontinuation from combination treatment period (as per the electronic case report form), interruptions of either drug before completion of the combination treatment period caused the mean duration of individual treatments to be shorter than that recorded for combination treatment.

23 patients $(26 \%)$ had treatment interruptions: one patient (1\%) had one interruption of pirfenidone alone, 16 patients (18\%) had 28 interruptions of nintedanib alone and six patients (7\%) had one interruption each of combination treatment (pirfenidone and nintedanib simultaneously interrupted); interruptions of combination treatment lasted a mean duration of 4.3 days. Mean length of all pirfenidone and nintedanib interruptions was 7.6 and 10.3 days, respectively.

88 patients (99\%) experienced 670 TEAEs; these were considered to be related to treatment in 74 patients (83\%; 418 treatment-related TEAEs) (table 2). Median (interquartile range) duration of TEAEs was 1.9 (0.3-11.3), $0.4(0.1-7.1)$ and 0.5 (0.1-6.4) weeks for TEAEs considered related to pirfenidone, nintedanib, and both pirfenidone and nintedanib, respectively. 67 patients (75\%) experienced gastrointestinal treatment-related TEAEs; the majority of gastrointestinal events were attributed by investigators to nintedanib alone (61 patients). Seven patients ( $8 \%$ ) experienced treatment-related photosensitivity or rash TEAEs (table 2).

TABLE 2 Summary of common treatment-emergent adverse events (TEAEs) and TEAEs leading to discontinuation (safety population"

$\begin{array}{cc}\text { Patients with at } & \text { Patients with at least one } \\ \text { least one } & \text { TEAE related to } \\ \text { TEAE } & \text { pirfenidone only }\end{array}$

\section{TEAEs occurring in $\geqslant 5 \%$ of patients} $\geqslant 1$ TEAE

$\geqslant 1$ treatment-related TEAE

Diarrhoea

Nausea

Vomiting

Decreased appetite

Fatigue

Dyspepsia

Headache

Weight decreased

Photosensitivity or rash

TEAEs

Abdominal pain upper

Dizziness

TEAEs leading to discontinuation $\geqslant 1 \mathrm{TEAE}$

$\geqslant 1$ treatment-related TEAE

Nausea

Diarrhoea

Fatigue

Weight decreased

Deep vein thrombosis

Epigastric discomfort

Malaise

Migraine

Vomiting

$\begin{array}{cc}88(99) & \\ 74(83) & 15(17) \\ 44(49) & 2(2) \\ 41(46) & 3(3) \\ 21(24) & 1(1) \\ 14(16) & 2(2) \\ 11(12) & 0 \\ 8(9) & 1(1) \\ 8(9) & 0 \\ 6(7) & 1(1) \\ 7(8) & 4(5) \\ 5(6) & \\ 5(6) & 1(1) \\ 13(15) & 0 \\ 11(12) & \\ 4(5) & \\ 4(5) & 0 \\ 2(2) & 0 \\ 2(2) & 0 \\ 1(1) & 0 \\ 1(1) & 0 \\ 1(1) & 0 \\ 1(1) & 0 \\ 1(1) & 0 \\ & 0\end{array}$

\section{Patients with at least one TEAE related to nintedanib only ${ }^{+}$}

Patients with at least one TEAE related to both pirfenidone and nintedanib ${ }^{+}$

Data are presented as $n(\%) .{ }^{\#}: \mathrm{n}=89 ;{ }^{\text {ๆ}}$ : each of the patients could have experienced one or more treatment-related TEAE, with the potential for different events to be related to different treatments; ${ }^{+}$: assessed by investigators for each therapy using their previous experience with pirfenidone and/or nintedanib, knowledge of the patient, the circumstances surrounding the event, and an evaluation of any potential alternative causes. 
13 patients (15\%) discontinued treatment due to TEAEs; these TEAEs were attributed to nintedanib alone in 10 patients $(11 \%)$, both pirfenidone and nintedanib in one patient $(1 \%)$, and were considered unrelated to either treatment in two patients (2\%), as assessed by the investigator (table 2). Discontinuation of combination treatment occurred throughout the trial and there was no clear pattern in the timing of early discontinuation over the treatment period (figure 3).

18 patients $(20 \%)$ experienced severe (Common Terminology Criteria for Adverse Events grade $\geqslant 3$ ) TEAEs; these were considered treatment-related in six patients (7\%) (table 3). 16 patients (18\%) experienced 18 serious TEAEs (table 3 and supplementary table S3); two patients (2\%) experienced one serious treatment-related TEAE each, both of which were attributed to nintedanib alone: one patient experienced a transient ischaemic attack but continued combination treatment without dose modification, and one patient experienced deep vein thrombosis and discontinued nintedanib but continued pirfenidone treatment. No fatal TEAEs were reported during the study.

Treatment-related hepatic TEAEs were reported in six patients (7\%); these were attributed to nintedanib alone in five patients (table 3$)$. All events were grade $1(n=4)$ or grade $2(n=1)$ elevations in liver enzymes, or grade 1 abnormal hepatic function $(n=1)$.

In an exploratory analysis of efficacy, mean \pm SE FVC $\%$ pred and DLCO \% pred declined by $0.8 \pm 0.6 \%$ and $1.4 \pm 0.8 \%$, respectively, from historical values to baseline and by $0.4 \pm 0.5 \%$ and $1.9 \pm 0.8 \%$, respectively, from baseline to week 24 in patients with available data (supplementary figure S1). An exploratory analysis of K-BILD from baseline to week 24 found that mean \pm SD total score decreased by $1.3 \pm 8.7$, and mean \pm SD individual subscores decreased by $2.4 \pm 14.3$ for the "psychological" domain, $0.7 \pm 15.9$ for the "breathlessness and activities" domain, and $3.1 \pm 17.0$ for the "chest symptoms" domain in patients completing 24 weeks of combination treatment; these changes might be due to chance, as the standard deviations are larger than the decreases (supplementary figure S2).

\section{Discussion}

This study found that combined treatment with pirfenidone $\left(1602-2403 \mathrm{mg} \cdot \mathrm{day}^{-1}\right)$ and nintedanib (200$300 \mathrm{mg} \cdot$ day $^{-1}$ ) for 24 weeks in 89 patients with IPF was completed by $78 \%$ of patients. The safety profile of combination treatment did not reveal a different pattern of TEAEs to that expected with either treatment alone. Most TEAEs affected the gastrointestinal system and were mild to moderate in severity. Furthermore, combination treatment taken for 24 weeks was not associated with increased risk of liver toxicity or photosensitivity. However, frequency of TEAEs (99\%) was high for a 6-month study, comparable with frequencies observed in the 12-month pirfenidone and nintedanib monotherapy trials $[18,24]$; although common gastrointestinal TEAEs occurred less frequently after 6 months of pirfenidone or nintedanib monotherapy in these studies $[25,26]$.

Results of this 24-week study are in line with those of the recent 12-week INJOURNEY trial in terms of overall TEAE frequency [21]. Both studies found that combination treatment with nintedanib and

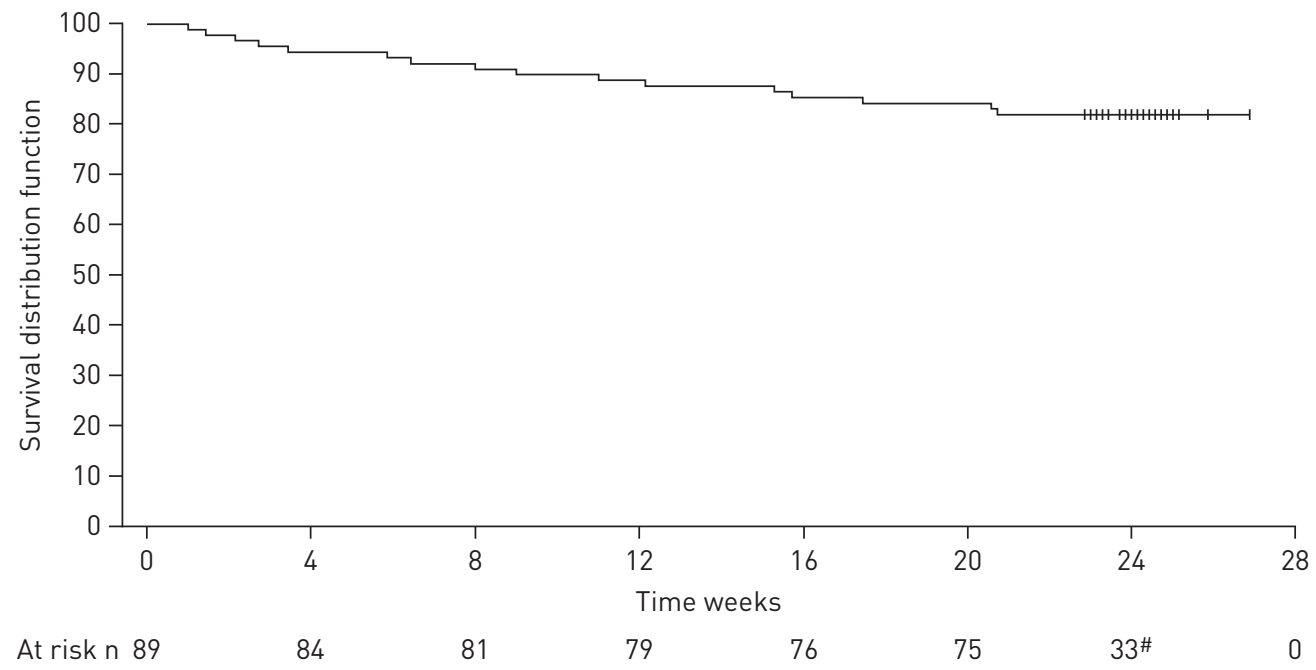

FIGURE 3 Time to discontinuation (safety population): includes time to early discontinuation or study completion. ${ }^{\#}$ : 40 patients completed the planned 24 weeks of combination treatment before study day 168 and were therefore censored on the Kaplan-Meier curve (represented by vertical lines). 
TABLE 3 Summary of severe, serious and hepatic treatment-emergent adverse events (TEAEs) (safety population")

\begin{tabular}{|c|c|c|}
\hline $\begin{array}{l}\text { Patients } \\
\text { with at } \\
\text { least one } \\
\text { TEAE }\end{array}$ & $\begin{array}{l}\text { Patients with at } \\
\text { least one TEAE } \\
\text { related to } \\
\text { pirfenidone only }\end{array}$ & $\begin{array}{l}\text { Patients with at } \\
\text { least one TEAE } \\
\text { related to } \\
\text { nintedanib only }\end{array}$ \\
\hline
\end{tabular}

Patients with at least one TEAE related to both pirfenidone and nintedanib ${ }^{+}$

\begin{tabular}{|c|c|c|c|c|}
\hline \multicolumn{5}{|l|}{ Severe TEAEs ${ }^{\S}$} \\
\hline$\geqslant 1 \mathrm{TEAE}$ & $18(20)$ & & & \\
\hline$\geqslant 1$ treatment-related TEAE & $6(7)$ & 0 & $5(6)$ & $1(1)$ \\
\hline Diarrhoea & $2(2)$ & 0 & $1(1)$ & $1(1)$ \\
\hline Nausea & $2(2)$ & 0 & $2(2)$ & 0 \\
\hline Fatigue & $1(1)$ & 0 & $1(1)$ & 0 \\
\hline Muscle spasms & $1(1)$ & 0 & $1(1)$ & 0 \\
\hline Weight decreased & $1(1)$ & 0 & $1(1)$ & 0 \\
\hline Deep vein thrombosis & $1(1)$ & 0 & $1(1)$ & 0 \\
\hline \multicolumn{5}{|l|}{ Serious TEAEs ${ }^{f}$} \\
\hline$\geqslant 1 \mathrm{TEAE}$ & $16(18)$ & & & \\
\hline$\geqslant 1$ treatment-related TEAE & $2(2)$ & 0 & $2(2)$ & 0 \\
\hline Transient ischaemic attack & $1(1)$ & 0 & $1(1)$ & 0 \\
\hline Deep vein thrombosis & $1(1)$ & 0 & $1(1)$ & 0 \\
\hline \multicolumn{5}{|l|}{ Hepatic TEAEs } \\
\hline$\geqslant 1 \mathrm{TEAE}$ & $7(8)$ & & & \\
\hline$\geqslant 1$ treatment-related TEAE & $6(7)$ & 0 & $5(6)$ & $1(1)$ \\
\hline GGT increased & $2(2)$ & 0 & $2(2)$ & 0 \\
\hline ALT increased & $2(2)$ & 0 & $2(2)$ & 0 \\
\hline AST increased & $1(1)$ & 0 & $1(1)$ & 0 \\
\hline $\begin{array}{l}\text { Aminotransferase } \\
\text { increased }\end{array}$ & $1(1)$ & 0 & $1(1)$ & 0 \\
\hline Blood ALP increased & $1(1)$ & 0 & $1(1)$ & 0 \\
\hline Hepatic function abnormal & $1(1)$ & 0 & $1(1)$ & 0 \\
\hline Elevated liver function test & $1(1)$ & 0 & $1(1)$ & 0 \\
\hline Elevated liver enzymes & $1(1)$ & 0 & 0 & $1(1)$ \\
\hline \multicolumn{5}{|c|}{ 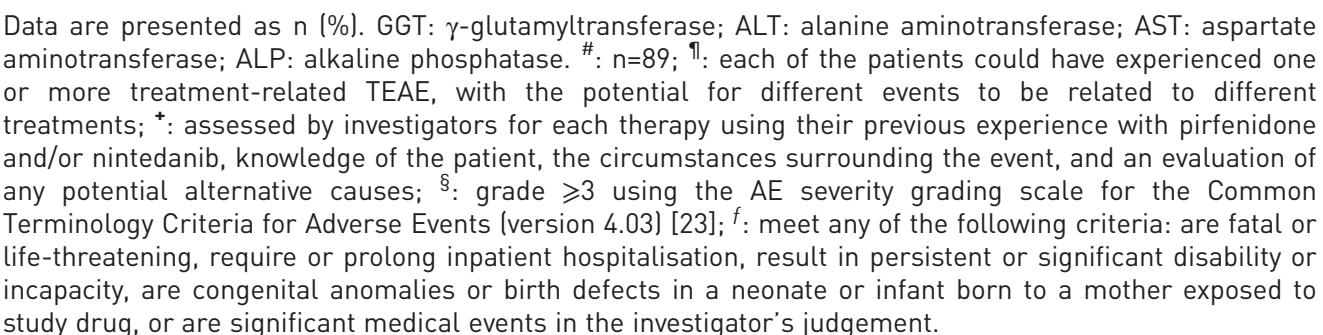 } \\
\hline
\end{tabular}

pirfenidone had a similar safety profile to that of pirfenidone or nintedanib monotherapy in terms of proportion of patients experiencing TEAEs and types of TEAEs reported. In the current study, patients were already tolerating a stable dose of pirfenidone prior to initiation of nintedanib, which may explain the higher incidence of TEAEs attributed to nintedanib versus pirfenidone by investigators. Similarly, the INJOURNEY trial (in which patients had already shown tolerability to nintedanib prior to initiating pirfenidone) found that more patients in the combination therapy group discontinued pirfenidone than nintedanib, although it should be noted that the study protocol recommended reducing pirfenidone dose before nintedanib dose for management of AEs other than diarrhoea [21].

In this study, treatment-related diarrhoea (49\% of patients) was reported more frequently than any diarrhoea TEAEs in previous studies of pirfenidone monotherapy (25\%) [24] and for combination treatment in the INJOURNEY trial (38\%) [21], but less frequently than any diarrhoea TEAEs in previous studies of nintedanib monotherapy (62\%) [18]. Moreover, treatment-related nausea (46\% of patients) was reported by more patients than any nausea TEAEs in previous studies of pirfenidone or nintedanib monotherapy (36\% and $24 \%$, respectively) $[18,24]$ and was comparable with nausea TEAEs reported in the INJOURNEY trial (42\%) [21]; however, the durations of the two studies were different, making such comparisons difficult. 
Although the frequency of some TEAEs was higher in this 24-week study than in studies of each therapy alone, discontinuation rates (18\% overall and $15 \%$ due to TEAEs) were numerically lower than those over 12 weeks in the INJOURNEY trial and over 52 weeks in the INPULSIS trials, and numerically similar to those over 52 weeks or more in the ASCEND and CAPACITY trials [9-11, 21]. Real-world data from the USA suggest that discontinuation rates for pirfenidone and nintedanib are higher in clinical practice $(24 \%$ for pirfenidone and $34 \%$ for nintedanib; mean follow-up of 8-9 months) than in clinical trials $[6,7,27]$. Therefore, it will be important to further investigate tolerability of combination treatment and develop strategies to help reduce the burden of gastrointestinal TEAEs. Current strategies to reduce nausea associated with pirfenidone include taking each dose with food; for reducing diarrhoea or nausea associated with nintedanib, ensuring adequate hydration and use of antidiarrhoea or antiemetic medications are recommended $[6,7,28,29]$. Dose adjustments are also recommended for management of AEs with both pirfenidone and nintedanib [6,7], and available data suggest that dose adjustments have a positive impact on treatment persistence [30] while maintaining the benefit of treatment on FVC decline [31]. In this study, $26 \%$ of patients had an interruption of combination treatment, but only $7 \%$ had both treatments simultaneously interrupted (for an average interruption length of $\sim 4$ days). This overall rate is similar to other clinical trial data for pirfenidone monotherapy (25\%) [31].

The exploratory efficacy analyses found that there was little decline in lung function during treatment with combined pirfenidone and nintedanib, which is similar to results in the INJOURNEY trial [21]. However, with the absence of a control group and larger sample size, no firm conclusions can be drawn regarding efficacy of combination treatment in patients with IPF. Quality of life, measured using the K-BILD questionnaire, did not worsen in patients who completed 24 weeks of treatment, which is in line with quality of life findings from the EuroQoL-5D questionnaire used in the INJOURNEY trial [21].

The benefits of combining therapies with different mechanisms of action have been demonstrated in a variety of chronic diseases, including chronic obstructive pulmonary disease, asthma and pulmonary arterial hypertension $(\mathrm{PAH})[32,33]$. Evidence from long-term studies in PAH has demonstrated that combination treatments targeting endothelin, nitric oxide and/or prostacyclin pathways have the potential to significantly delay disease progression [33]. Indeed, the treatment strategy for PAH is shifting towards use of first-line combination therapy [32]. Results of the current study suggest that combination treatment with pirfenidone and nintedanib could provide a viable future option for patients with IPF. However, combination therapy might not be suitable for all patients, and further research will be necessary to study long-term benefits and risks of combination treatment in a controlled study with treatment duration of $>6$ months.

Limitations of this study include the lack of a control or comparator group and small sample size, which are due to the exploratory nature of the study. Furthermore, only patients who tolerated pirfenidone were included, which may introduce a bias towards patients less likely to experience TEAEs, and patients could have treatment interrupted for $<28$ consecutive days and still be considered to have completed 24 weeks of combination treatment. Thus, tolerance to treatment in the real-world might be less than that reflected in the completion rate. Given that pirfenidone and nintedanib treatment can result in similar AEs $[9,10,18]$, there may have been uncertainty regarding whether some TEAEs were related specifically to pirfenidone or nintedanib. As nintedanib was added to stable pirfenidone treatment in this study, conclusions cannot be drawn for pirfenidone added to stable nintedanib treatment or when both treatments are started simultaneously. Although patients with more advanced IPF (FVC $\%$ pred $<50 \%$ and DLCO $\%$ pred $<30 \%$ ) might also be expected to benefit from combination treatment, these patients were excluded from the trial. However, eligibility criteria were similar to the pivotal trials [9-11], allowing comparison of safety data between this study and clinical trials of pirfenidone monotherapy. Finally, changes in FVC, DLCO and K-BILD were tested as exploratory end-points, and the study was not designed to formally assess efficacy of combination treatment; historical values were used to compare pre-treatment changes in FVC and DLCO.

\section{Conclusions}

Combined use of pirfenidone and nintedanib for 24 weeks was tolerated by the majority of patients with IPF, and associated with similar types of TEAEs and discontinuation rates expected with either treatment alone. Results of this trial add to the limited safety data of combination treatment taken for $>3$ months, and encourage further study to determine the efficacy and safety of pirfenidone and nintedanib taken as combination treatment versus monotherapy in patients with IPF.

Acknowledgements: Medical writing support was provided by Rebekah Waters on behalf of CMC AFFINITY, a division of Complete Medical Communications Ltd, Manchester, UK, funded by F. Hoffmann-La Roche, Ltd. The authors would like to thank the patients that participated in this trial and the research teams that facilitated the trial. The independent data monitoring committee members for this study were Marilyn K. Glassberg (Chair; University of Miami, Miami, FL, USA), Ulrich Costabel (Ruhrlandklinik, Essen, Germany) and Diethelm Messinger (PROMETRIS GmbH, Mannheim, Germany). 
List of investigators: Charlene D. Fell (Calgary, Canada), Shane Shapera (Toronto, Canada), Saher B. Shaker (Hellerup, Denmark), Vincent Cottin (Bron, France), Stephane Jouneau (Rennes, France), Hilario Nunes (Bobigny, France), Francesco Tommaso Bonella (Essen, Germany), Dirk Koschel (Coswig, Germany), Philipp Markart (Fulda, Germany), Carlo Albera (Orbassano, Italy), Alberto Pesci (Monza, Italy), Paola Rottoli (Siena, Italy), Laura Tavanti (Cisanello, Italy), Marcel Veltkamp (Nieuwegein, The Netherlands), Marlies Wijsenbeek (Rotterdam, The Netherlands), Orlando Acosta (La Laguna, Spain), Elena Bollo (León, Spain), Alvaro Casanova Espinosa (Madrid, Spain), Juan Gustavo (Valencia, Spain), Maria Molina-Molina (Barcelona, Spain), Jose A. Rodriguez-Portal (Seville, Spain), Claudia Valenzuela (Madrid, Spain), John Belperio (Los Angeles, CA, USA), John Butler (Portland, OR, USA), Sakshi Dua (New York, NY, USA), Kevin R. Flaherty (Ann Arbor, MI, USA), Todd Horiuchi (Sarasota, FL, USA), J. Terrill Huggins (Charleston, SC, USA), Gaurav Khanna (Cincinnati, OH, USA), Christopher King (Falls Church, VA, USA), Andrew Labelle (Chesterfield, MO, USA), Lisa H. Lancaster (Nashville, TN, USA), Joshua Mooney (Stanford, CA, USA), Lee Morrow (Omaha, NE, USA), Nina Patel (New York, NY, USA), Murali Ramaswamy (Greensboro, NC, USA), Robert Sussman (Springfield, NJ, USA), Joseph Zibrak (Boston, MA, USA).

Author contributions: All authors were involved in the design of this study and the interpretation of study results, contributed to the manuscript from the outset, and read and approved the final draft. All authors vouch for the accuracy of the content included in the final manuscript.

Conflict of interest: K.R. Flaherty has received consultant and research support fees from F. Hoffmann-La Roche/ Genentech and Boehringer Ingelheim, consultant fees from FibroGen, Veracyte, Aeolus, PharmaKea, Sanofi-Genzyme and Celgene, and research support fees from Afferent Pharmaceuticals. C.D. Fell has received consultant and research support fees from F. Hoffmann-La Roche Canada and consultant fees from Boehringer Ingelheim. J.T. Huggins has received consultant and research support fees from F. Hoffmann-La Roche/Genentech and Boehringer Ingelheim, and consultant fees from Gilead and iBIOS. H. Nunes has received consultant and research support fees from F. Hoffmann-La Roche/Genentech and Boehringer Ingelheim, and has been an investigator of clinical trials for Sanof and Gilead. R. Sussman has received research support and speaker and consultation fees from F. Hoffmann-La Roche/ Genentech and Boehringer Ingelheim, and research support from Gilead. C. Valenzuela has received speaker and consultation fees from F. Hoffmann-La Roche and Boehringer Ingelheim. J.L. Stauffer is an employee of Genentech and a former employee of FibroGen, and holds shares from both. F. Gilberg is an employee of F. Hoffmann-La Roche. M. Bengus is an employee of F. Hoffmann-La Roche. M. Wijsenbeek has received speaker and consultation fees and unrestricted research grants from F. Hoffmann-La Roche and Boehringer Ingelheim, and consultancy fees from Galapagos; all fees and grants were paid to her institution.

Support statement: This trial was funded by F. Hoffmann-La Roche, Ltd. Funding information for this article has been deposited with the Crossref Funder Registry.

\section{References}

1 Ley B, Collard HR, King TE Jr. Clinical course and prediction of survival in idiopathic pulmonary fibrosis. Am J Respir Crit Care Med 2011; 183: 431-440.

2 Raghu G, Collard HR, Egan JJ, et al. An official ATS/ERS/JRS/ALAT statement: idiopathic pulmonary fibrosis: evidence-based guidelines for diagnosis and management. Am J Respir Crit Care Med 2011; 183: 788-824.

3 American Cancer Society. Cancer Facts \& Figures 2017. 2017. www.cancer.org/content/dam/cancer-org/research/ cancer-facts-and-statistics/annual-cancer-facts-and-figures/2017/cancer-facts-and-figures-2017.pdf Date last accessed: January 15, 2018.

4 Nathan SD, Shlobin OA, Weir N, et al. Long-term course and prognosis of idiopathic pulmonary fibrosis in the new millennium. Chest 2011; 140: 221-229.

$5 \quad$ Siegel RL, Miller KD, Jemal A. Cancer statistics, 2018. CA Cancer J Clin 2018; 68: 7-30.

6 Food and Drug Administration. Highlights of Prescribing Information Ofev. 2017. www.accessdata.fda.gov/ drugsatfda_docs/label/2017/205832s004lbl.pdf Date last accessed: March 29, 2018.

7 Genentech. Product label: Full prescribing information - Esbriet. 2017. www.gene.com/download/pdf/esbriet prescribing.pdf Date last accessed: January 26, 2018.

8 Raghu G, Rochwerg B, Zhang Y, et al. An official ATS/ERS/JRS/ALAT clinical practice guideline: treatment of idiopathic pulmonary fibrosis. An update of the 2011 clinical practice guideline. Am J Respir Crit Care Med 2015; 192: e3-e19.

9 Noble PW, Albera C, Bradford WZ, et al. Pirfenidone in patients with idiopathic pulmonary fibrosis (CAPACITY): two randomised trials. Lancet 2011; 377: 1760-1769.

10 King TE Jr, Bradford WZ, Castro-Bernardini S, et al. A phase 3 trial of pirfenidone in patients with idiopathic pulmonary fibrosis. N Engl J Med 2014; 370: 2083-2092.

11 Richeldi L, du Bois RM, Raghu G, et al. Efficacy and safety of nintedanib in idiopathic pulmonary fibrosis. $N$ Engl J Med 2014; 370: 2071-2082.

12 Didiasova $M$, Singh R, Wilhelm J, et al. Pirfenidone exerts antifibrotic effects through inhibition of GLI transcription factors. FASEB J 2017; 31: 1916-1928.

13 European Medicines Agency. Summary of Product Characteristics - Esbriet (pirfenidone). 2017. www.ema.europa. eu/docs/en_GB/document_library/EPAR_-_Product_Information/human/002154/WC500103049.pdf Date last accessed: March 27, 2018.

14 European Medicines Agency. Summary of Product Characteristics - Ofev (nintedanib). 2017. www.ema.europa.eu/ docs/en_GB/document_library/EPAR_-_Product_Information/human/003821/WC500182474.pdf Date last accessed: March 27, 2018.

15 Lehtonen ST, Veijola A, Karvonen H, et al. Pirfenidone and nintedanib modulate properties of fibroblasts and myofibroblasts in idiopathic pulmonary fibrosis. Respir Res 2016; 17: 14.

16 King TE Jr, Pardo A, Selman M. Idiopathic pulmonary fibrosis. Lancet 2011; 378: 1949-1961.

17 Knuppel L, Ishikawa Y, Aichler M, et al. A novel antifibrotic mechanism of nintedanib and pirfenidone. Inhibition of collagen fibril assembly. Am J Respir Cell Mol Biol 2017; 57: 77-90. 
18 Richeldi L, Cottin V, du Bois RM, et al. Nintedanib in patients with idiopathic pulmonary fibrosis: combined evidence from the TOMORROW and INPULSIS trials. Respir Med 2016; 113: 74-79.

19 Ogura T, Taniguchi H, Azuma A, et al. Safety and pharmacokinetics of nintedanib and pirfenidone in idiopathic pulmonary fibrosis. Eur Respir J 2015; 45: 1382-1392.

20 Taniguchi $\mathrm{H}$, Ogura $\mathrm{T}$, Inoue $\mathrm{Y}$, et al. Long-term safety of combination therapy with nintedanib and pirfenidone in Japanese patients with IPF. Eur Respir J 2016; 48: PA2089.

21 Vancheri C, Kreuter M, Richeldi L, et al. Nintedanib with add-on pirfenidone in idiopathic pulmonary fibrosis: results of the INJOURNEY trial. Am J Respir Crit Care Med 2018; 197: 356-363.

22 Patel AS, Siegert RJ, Brignall K, et al. The development and validation of the King's Brief Interstitial Lung Disease (K-BILD) health status questionnaire. Thorax 2012; 67: 804-810.

23 US Dept of Health and Human Services, Common Terminology Criteria for Adverse Events (CTCAE). Version 4.0. 2010. https://evs.nci.nih.gov/ftp1/CTCAE/CTCAE_4.03_2010-06-14_QuickReference_5×7.pdf Date last accessed: January 30, 2018.

24 Noble PW, Albera C, Bradford WZ, et al. Pirfenidone for idiopathic pulmonary fibrosis: analysis of pooled data from three multinational phase 3 trials. Eur Respir J 2016; 47: 243-253.

25 Corte T, Bonella F, Crestani B, et al. Safety, tolerability and appropriate use of nintedanib in idiopathic pulmonary fibrosis. Respir Res 2015; 16: 116.

26 Lancaster LH, de Andrade J, Zibrak JD, et al. Pirfenidone safety and adverse event management in idiopathic pulmonary fibrosis. Eur Respir Rev 2017; 26: 170057.

27 Lalla D, Bengston L, Raimundo K, et al. Pirfenidone and nintedanib compliance and persistence in a real world setting [poster presentation at the American Thoracic Society Conference 2017]. Am J Respir Crit Care Med 2017; 195: A5351.

28 Costabel U, Bendstrup E, Cottin V, et al. Pirfenidone in idiopathic pulmonary fibrosis: expert panel discussion on the management of drug-related adverse events. Adv Ther 2014; 31: 375-391.

29 Cottin V. The safety and tolerability of nintedanib in the treatment of idiopathic pulmonary fibrosis. Expert Opin Drug Saf 2017; 16: 857-865.

30 Cottin V, Koschel D, Gunther A, et al. Long-term safety of pirfenidone in a real-world setting: final results from the prospective, observational PASSPORT registry [poster presentation at the European Respiratory Society International Congress 2017]. Eur Respir J 2017; 50: Suppl. 61, PA2806.

31 Nathan SD, Lancaster L, Albera C, et al. Dose modifications and dose intensity during treatment with pirfenidone [oral presentation at the European Respiratory Society International Congress 2016]. Eur Respir J 2016; 48: Suppl. 60, OA1764.

32 Wuyts WA, Antoniou KM, Borensztain K, et al. Combination therapy: the future of management for idiopathic pulmonary fibrosis? Lancet Respir Med 2014; 2: 933-942.

33 Sitbon O, Gaine S. Beyond a single pathway: combination therapy in pulmonary arterial hypertension. Eur Respir Rev 2016; 25: 408-417. 